> L'hémostase est un processus complexe qui implique de nombreux acteurs cellulaires et moléculaires. En pathologie, les thromboses d'une part, et les pathologies hémorragiques constitutionnelles dominées par l'hémophilie d'autre part, ont bénéficié ces dernières années du développement d'anticorps thérapeutiques qui révolutionnent aujourd'hui la prise en charge des malades. <

\section{Les anticorps thérapeutiques anti-thrombotiques}

\section{L'abciximab, le précurseur}

L'abciximab est le premier anticorps ayant été développé pour le traitement de thromboses. C'est le fragment Fab d'un anticorps monoclonal (7£3) humanisé, qui inhibe l'agrégation plaquettaire en se fixant de façon réversible aux récepteurs GPIIb/IIla, empêchant ainsi la fixation plaquettaire du fibrinogène et du facteur Willebrand (vWF). L'abciximab se lie aussi à l'intégrine CD1lb/CD18 (ou Mac-1) présente sur les monocytes activés et au récepteur de la vitronectine exprimé par l'endothélium $\left(\alpha_{v} \beta_{3}\right)$. L'ensemble de ces propriétés induit chez le patient traité un état comparable à celui d'un malade ayant une thrombasthénie de Glanzmann ${ }^{1}$. Son efficacité en clinique a été validée dans le syndrome coronarien aigu (SCA) grâce aux études EPIC, EPILOG [1], et CAPTURE [2], et, combiné à l'héparine et à l'acide acétylsalicylique, il prévient les complications cardiaques ischémiques chez les patients subissant une intervention coronarienne percutanée et ayant un angor instable. Bien que moins utilisé aujourd'hui, cet anticorps reste très utile en sauvetage en cas de thrombose quand les antiplaquettaires actuels (prasugrel, ticagrelor, notamment) sont insuffisamment efficaces.

${ }^{1}$ La maladie de Glanzmann (ou thrombasthénie de Glanzmann) est une maladie hémorragique héréditaire rare, causée par un défaut d'agrégation plaquettaire. La dysfonction est causée par une anomalie génétique qui touche les gènes qui codent les glycoprotéines IIb/IIla (qui constituent le récepteur du fibrinogène).

\section{Les anticorps thérapeutiques en hémostase D'hier, d'aujourd'hui et de demain...}

\section{Yves Gruel ${ }^{1,2}$, Claire Kizlik-Masson ${ }^{3}$, Peter Lenting ${ }^{3}$}

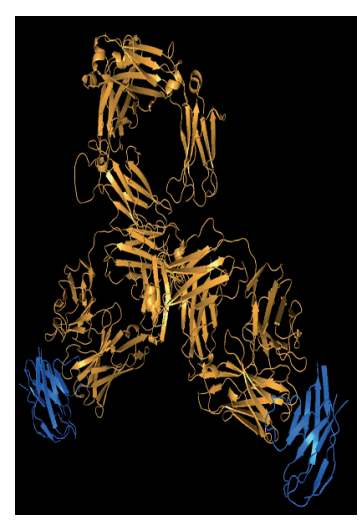

${ }^{1}$ EA 7501, GICC, Université François Rabelais, Tours, France. 2Laboratoire d'hématologiehémostase, CHU Tours, France. ${ }^{3}$ Inserm, UMR-S 1176, Université Paris-Sud, Université ParisSaclay, 94276 Le KremlinBicêtre, France gruel@med.univ-tours.fr

Les nouveaux anticorps anti-thrombotiques D'autres anticorps ont été développés récemment et la plupart ne sont pas encore utilisés en thérapeutique. Ils ciblent soit l'hémostase primaire, notamment l'interaction Willebrand/GPIb (caplacizumab) ou la glycoprotéine VI (GPVI) (ACT017), soit la coagulation, en inhibant les facteurs XI et XII.

\section{Le caplacizumab, un nanobody efficace dans le PTT}

Le purpura thrombotique thrombocytopénique (PTT) est une microangiopathie thrombotique rare, associant une anémie hémolytique mécanique, une thrombopénie sévère, et une ischémie viscérale due à des microthrombus riches en plaquettes et disséminés. Le PTT résulte souvent d'un déficit fonctionnel sévère en ADAMTS13 (A disintegrin and metalloprotease with thrombospondin type 1 repeats -13$)^{2}$, principalement dû à des auto-anticorps anti-ADAMTS13, et très rarement à des mutations du gène ADAMTS13, entraînant l'accumulation de multimères de facteur Willebrand (VWF) de très grande taille, hyperadhésifs pour les plaquettes, et induisant spontanément la formation de microthrombus dans les petits vaisseaux.

Le caplacizumab est un nanobody correspondant au domaine variable VHH d'un anticorps mono-chaîne de camélidé dirigé contre le domaine Al du vWF. II inhibe spécifiquement l'interaction du vWF avec la GPIb plaquettaire dans les conditions de forces de cisaillement élevées (lesquelles prédominent dans les petits vaisseaux). Son efficacité à prévenir les thromboses, ainsi que sa sécurité d'emploi, ont été initialement démontrées chez le babouin dans un modèle de PTT acquis. Plus récem-

\footnotetext{
${ }^{2}$ La protéase spécifique du clivage du facteur von Willebrand.
} 
ment, le caplacizumab a été évalué dans 2 essais, TITAN [3] et HERCULES [4]. Dans ce dernier, 145 malades randomisés (1:1) ont reçu en plus d'un échange plasmatique quotidien, un placebo ou un bolus intraveineux de $10 \mathrm{mg}$ de caplacizumab, suivi d'une injection par voie sous-cutanée de la même dose du nanobody ou de placebo pendant 30 jours. Selon la réponse clinique obtenue, le traitement pouvait être prolongé. Une réduction du temps de correction de la numération plaquettaire, de la mortalité due au PTT, des accidents thrombotiques, et une meilleure prévention des rechutes ont été observées quand le traitement avec le caplacizumab était prolongé. Ces résultats expliquent que le caplacizumab (Cablivi ${ }^{\circledR}$ ) ait été approuvé en Europe pour le traitement du PTT en 2018.

\section{ACT017, un Fab humanisé anti-GPVI}

La glycoprotéine GPVI est un récepteur plaquettaire spécifique du collagène qui est primordial pour la formation des thromboses artérielles. Neutraliser la GPVI est donc susceptible d'inhiber l'agrégation plaquettaire sans induire d'effets trop délétères sur l'hémostase physiologique. Ces données expliquent le fait que Martine Jandrot-Perrus et son équipe aient développé un anticorps Fab humanisé bloquant la GPVI, dénommé ACT017, sélectionné selon ses propriétés structurales et fonctionnelles [5]. L'ACT017 a une affinité et une spécificité élevées pour la GPVI et il inhibe ex vivo, de façon dose-dépendante, l'agrégation plaquettaire induite par le collagène chez le macaque sans induire de thrombopénie. Une étude de phase I chez l'homme réalisée en 2018 a permis de confirmer la sécurité d'emploi de ce Fab anti-GPVI et un essai de phase II est programmé.

\section{F7 inhibe le facteur XIla sans majorer le saignement}

Plusieurs études ont souligné l'intérêt d'inhiber la voie dite «contact » de la coagulation (activée non physiologiquement par le contact du sang avec une surface mouillable) et notamment les facteurs XI et XII afin d'exercer un effet anti-thrombotique significatif sans risque hémorragique.

Ainsi, Thomas Renné a émis l'hypothèse qu'en inhibant sélectivement le facteur XIIa lors d'une assistance circulatoire, l'on pouvait éviter les thromboses dans le circuit, avec un risque de saignement plus faible qu'avec I'héparine. II a donc développé 3F7, un anticorps (IgG4) se fixant spécifiquement sur le site actif du facteur XIla, anticorps avec lequel il a confirmé cette hypothèse dans un modèle animal de circulation extracorporelle [6].

D'autres anticorps spécifiques du facteur XII (AB052) ou du facteur XI (aXIMAb) ont aussi été développés et sont actuellement évalués, notamment dans des modèles d'assistance circulatoire $[7,8]$.

\section{Les anticorps thérapeutiques antihémorragiques}

L'hémophilie est la pathologie hémorragique constitutionnelle qui a le plus bénéficié ces dernières années du développement d'anticorps thérapeutiques, avec deux approches différentes: I'une vise à mimer, avec un anticorps, certains des effets physiologiques du facteur VIII nécessaires pour générer la thrombine; l'autre a pour but de développer des anticorps inhibant les anticoagulants naturels comme le TFPI (tissue factor pathway inhibitor). D'autres anticorps ont également été produits, en dehors de l'hémophilie, pour servir d'antidotes à des antithrombotiques, notamment le dabigatran et le ticagrelor.

\section{Les anticorps thérapeutiques dans l'hémophilie}

\section{L'émicizumab, un anticorps mimant le facteur VIII}

L'hémophilie A est une maladie traitée par des injections répétées de facteur VIII qui sont contraignantes et induisent l'apparition d'anticorps neutralisants dans plus de $30 \%$ des cas. Dans ce contexte, un laboratoire japonais a développé un anticorps chimérique humanisé bispécifique, l'émicizumab, une IgG4 qui se fixe spécifiquement aux facteurs $I X a$ et $X$ de la coagulation [9]. II mime donc la capacité du facteur VIII à rapprocher l'enzyme (le IXa) et son substrat (le X), et permet ainsi de restituer une production de thrombine permettant à un hémophile sévère, même s'il a développé un inhibiteur anti-FVIII, d'être bien protégé vis-à-vis de saignements articulaires ou autres. Sa longue demi-vie d'élimination permet de traiter un malade avec une seule injection par voie sous-cutanée par semaine et, comme son efficacité a été validée chez l'enfant et l'adulte [10, 11], l'émicizumab (Hemlibra ${ }^{\circledR}$ ) a obtenu une AMM en $2018 \mathrm{chez}$ les patients ayant développé un anticorps anti-FVIII. De rares malades traités peuvent aussi développer des anticorps spécifiques de l'émicizumab (ADA, pour « antidrug antibody »), mais très souvent sans conséquences. L'action procoagulante de ce médicament n'est pas régulée in vivo comme celle du facteur VIII [12], et des thromboses ou des microangiopathies thrombotiques ont été observées dans quelques cas traités, mais ayant reçu des doses élevées de FEIBA, un concentré extrait du plasma et apportant des formes activées et non activées de facteurs II, VII, IX, et X. Cependant, le facteur VII activé recombinant peut être associé à l'émicizumab sans risque.

Compte tenu de son efficacité et sa simplicité d'utilisation, l'Hemlibra ${ }^{\circledR}$ est en passe de devenir dans les pays riches l'un des médicaments les plus prescrits pour une prophylaxie chez l'hémophile A sévère.

\section{Les anticorps inhibiteurs du TFPI}

Le TFPI (tissue factor pathway inhibitor) est un inhibiteur plasmatique synthétisé par les cellules endothéliales mais présent aussi dans les plaquettes et les monocytes. Il régule l'initiation de la coagulation en neutralisant le facteur VIla, et le complexe prothrombinase en inhibant le facteur Xa [13]. Différentes observations ont suggéré qu'en inhibant le TFPI, I'on pouvait restituer une génération de thrombine et une 


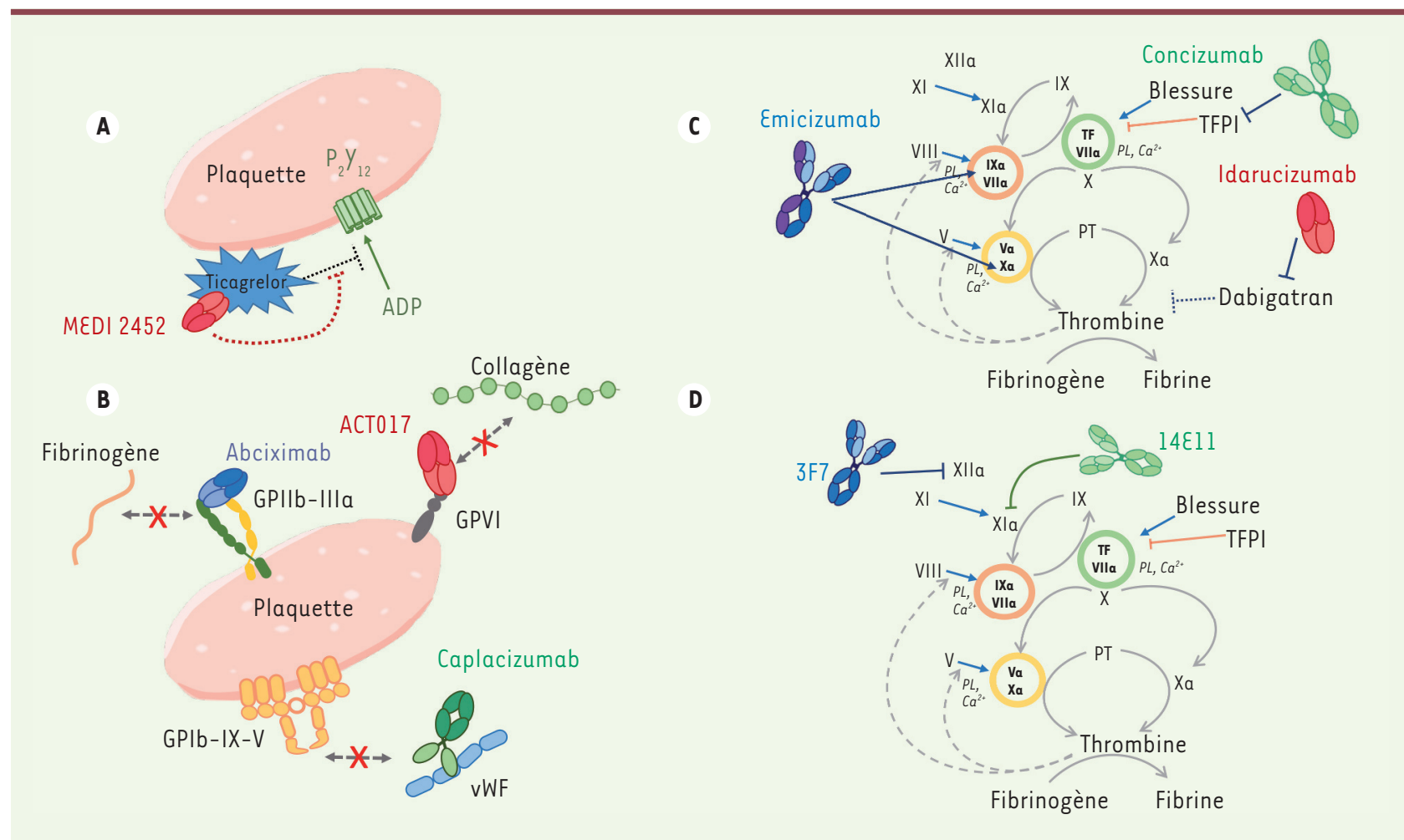

Figure 1. Cibles exprimées par les plaquettes (A, B) ou les facteurs de la coagulation d'anticorps thérapeutiques (ou fragments d'anticorps) ayant une activité antihémorragique $(A, C)$ ou antithrombotique $(B, D)$.

hémostase suffisante pour prévenir la survenue de saignements chez un hémophile sévère [14], qu'il soit déficitaire en facteur VIII ou en facteur IX. Plusieurs anticorps neutralisants le TFPI ont donc été développés. Le plus avancé en termes d'évaluation est le concizumab, une IgG4 fixant le domaine K2 du TFPI (qui inhibe le facteur Xa). Une étude de phase I réalisée chez 24 hémophiles $A$ sévères a récemment confirmé l'amélioration de la génération de thrombine associée à la diminution du taux de TFPI libre par ce traitement, d'une façon dose-dépendante. Aucun effet indésirable n'a été rapporté, mais une augmentation des taux de $\mathrm{D}$-dimères et de fragment $\mathrm{Fl}+2$ de la prothrombine peut faire craindre un risque de thrombose [15].

Une étude réalisée avec le PF-06741086, une IgGl spécifique du domaine K2 du TFPI [16] a conduit à des résultats comparables. Un autre anticorps anti-TFPI, le mAb2F22, se fixe exclusivement au domaine Kl du TFPI et interfère avec l'inhibition du facteur VIla, mais aussi du facteur $\mathrm{Xa}$, en restaurant la formation du caillot [17].

Enfin, un anticorps de sous-classe IgG2 (BAY 1093884) qui se fixe à la fois aux domaines $\mathrm{Kl}$ et K2 du TFPI, est en cours d'étude [18].

\section{Mab508, un anticorps protégeant le facteur Willebrand}

Trente à $50 \%$ des malades sous assistance cardio-circulatoire (LVAD [left ventricular assist device] ou ECMO [extra-corporeal membrane oxygenation]) présentent des saignements [19] qui sont favorisés par la perte des fonctions hémostatiques du vWF dégradé de façon majorée par ADAMTS13. Sophie Susen et Peter Lenting ont donc développé un anticorps anti-vWF (Mab508) qui bloque spécifiquement cette dégradation [20]. Cet effet inhibiteur n'excède pas $50 \%$, de façon à éviter une microangiopathie thrombotique. Cet anticorps est actuellement évalué dans des tests précliniques.

\section{Les anticorps antidotes de médicaments antithrombotiques}

\section{L'idarucizumab, antidote du dabigatran, une anti- thrombine oral}

Le dabigatran, premier anticoagulant oral direct utilisé chez l'homme, inhibe directement la thrombine libre ou liée au caillot de fibrine. Point essentiel, il est exclusivement éliminé par le rein. Souvent prescrit chez le sujet âgé, il expose donc à un risque élevé de surdosage et d'hémorragie sévère en cas d'insuffisance rénale. Un fragment Fab humanisé (l'idarucizumab) qui se fixe au dabigatran, dans un rapport stœchiométrique $1: 1$, et inhibe rapidement son activité anticoagulante, a été développé [21]. Son affinité pour le dabigatran est 350 fois plus élevée que celle du dabigatran pour la thrombine (avec des Kd respectives de $2 \mathrm{pM}$ et 0,7 nM). Après des études précliniques, l'efficacité et la sécurité d'emploi de l'idarucizumab ont été validées chez I'homme [22, 23], et ce médicament a obtenu une AMM en Europe en 2016. 


\section{Le PB2452, antidote du ticagrelor, un puissant antiplaquettaire}

Le ticagrelor est un antiplaquettaire puissant qui inhibe la signalisation induite par la fixation de l'ADP à l'un de ses récepteurs purinergiques, P2y12. Prescrit dans le syndrome coronarien aigu, il a une demi-vie d'élimination courte, mais en cas de saignement sévère chez un malade traité, le seul recours reste la transfusion de plaquettes, qui s'avère malheureusement souvent inefficace, le métabolite du ticagrelor inhibant aussi les plaquettes fraichement produites dans la moelle osseuse [24]. Un anticorps antidote spécifique a donc été développé par Buchanan et al. qui, après avoir criblé une bibliothèque de phages exprimant des Fab (phage display), ont sélectionné deux phages ayant une affinité significative pour le ticagrelor ou son métabolite actif [25]. Le médicament élaboré, MEDI2452, est un Fab avec une affinité de $20 \mathrm{pM} / \mathrm{L}$ pour le ticagrelor et son métabolite actif, 100 fois plus élevée que celle du ticagrelor pour P2y12 (2 nM/L). Les résultats d'une étude de phase I finalisée fin 2018 sont en attente.

\section{Conclusion}

L'apport récent d'anticorps thérapeutiques en hémostase a permis des progrès importants non seulement dans l'hémophilie, mais aussi dans certaines situations associées à des thromboses. Plusieurs molécules nouvelles sont en cours d'évaluation, notamment des nanobodies. L'un d'entre eux, spécifique du vWF, a récemment été fusionné au facteur VIII. Avec cette nouvelle et prometteuse protéine de fusion, la demivie d'élimination du facteur est majorée et la réponse immunitaire et indésirable vis-à-vis du facteur VIII est diminuée, chez la souris [26]. $\diamond$ Therapeutic antibodies in hemostasis. From the past to the future

\section{LIENS D'INTÉRÊT}

Les auteurs déclarent n'avoir aucun lien d'intérêt concernant les données publiées dans cet article.

\section{RÉFÉRENCES}

1. Ellis SG, Lincoff AM, Miller D, et al. Reduction in complications of angioplasty with abciximab occurs largely independently of baseline lesion morphology. EPIC and EPILOG Investigators. Evaluation of $7 \varepsilon 3$ for the prevention of ischemic complications. Evaluation of PTCA to improve long-term outcome with abciximab GPIIb/IIla receptor blockade.1998; 32 : 1619-23.

2. Randomised placebo-controlled trial of abciximab before and during coronary intervention in refractory unstable angina: the CAPTURE Study. Lancet 1997 ; 349 : 1429-35.

3. Peyvandi F, Scully M, Kremer Hovinga JA, et al. Caplacizumab for acquired thrombotic thrombocytopenic purpura. N Engl I Med 2016 ; 374 : 511-22.

4. Dane K, Chaturvedi S. Beyond plasma exchange: novel therapies for thrombotic thrombocytopenic purpura. Hematology Am Soc Hematol Educ Program 2018 ; 2018 : 539-47.

5. Lebozec K, Jandrot-Perrus M, Avenard G, et al. Design, development and characterization of ACT017, a humanized Fab that blocks platelet's glycoprotein VI function without causing bleeding risks. MAbs. 2017 ; 9 : 945-58.

6. Larsson M, Rayzman V, Nolte MW, et al. A factor XIla inhibitory antibody provides thromboprotection in extracorporeal circulation without increasing bleeding risk. Sci Transl Med $2014 ; 6: 222$ ral7.
7. Wallisch M, Tucker El, Lorentz CU, et al. The anti-factor XII antibody AB052 is antithrombotic without hemostatic impairment in a primate model of extracorporeal membrane oxygenation. Blood 2017 ; $130: 236$.

8. Tucker El, Marzec UM, White TC, et al. Prevention of vascular graft occlusion and thrombus-associated thrombin generation by inhibition of factor $\mathrm{XI}$. Blood $2009 ; 113$ : 936-44.

9. Kitazawa T, Igawa T, Sampei Z, et al. A bispecific antibody to factors IXa and $X$ restores factor VIII hemostatic activity in a hemophilia A model. Nat Med $2012 ; 18: 1570-4$.

10. Oldenburg J, Mahlangu JN, Kim B, et al. Emicizumab prophylaxis in hemophilia A with inhibitors. N Engl J Med 2017 ; 377 : 809-18.

11. Young G, Callaghan M, Dunn A, et al. Emicizumab for hemophilia A with factor VIII inhibitors. Expert Rev Hematol 2018 ; 11 : 835-46.

12. Lenting PJ, Denis CV, Christophe OD. Emicizumab, a bispecific antibody recognizing coagulation factors IX and X: how does it actually compare to factor VIII? 2017 ; 130 : 2463-8.

13. Mast AE. Tissue factor pathway inhibitor: multiple anticoagulant activities for a single protein. Arterioscler Thromb Vasc Biol 2016 ; 36 : 9-14.

14. Hilden I, Lauritzen B, Sorensen BB, et al. Hemostatic effect of a monoclonal antibody mAb 2021 blocking the interaction between FXa and TFPI in a rabbit hemophilia model. Blood 2012 ; 119 : 5871-8.

15. Eichler H, Angchaisuksiri P, Kavakli K, et al. A randomized trial of safety, pharmacokinetics and pharmacodynamics of concizumab in people with hemophilia A. J Thromb Haemost 2018 ; 16 : 2184-95.

16. Cardinal M, Kantaridis C, Zhu T, et al. A first-in-human study of the safety, tolerability, pharmacokinetics and pharmacodynamics of PF-06741086, an anti-tissue factor pathway inhibitor $\mathrm{mAb}$, in healthy volunteers. J Thromb Haemost 2018 ; 16 : 1722-31.

17. Augustsson C, Svensson A, Kjaer B, et al. Factor Xa and VIla inhibition by tissue factor pathway inhibitor is prevented by a monoclonal antibody to its Kunitz-1 domain. J Thromb Haemost 2018 ; $16: 893-904$.

18. Gu JM, Zhao XY, Schwarz T, et al. Mechanistic modeling of the pharmacodynamic and pharmacokinetic relationship of tissue factor pathway inhibitor-neutralizing antibody (BAY 1093884) in cynomolgus monkeys. AAPS J 2017 ; 19 : 1186-95.

19. Susen $S$, Rauch A, Van Belle $\varepsilon$, et al. Circulatory support devices: fundamental aspects and clinical management of bleeding and thrombosis. J Thromb Haemost 2015 ; 13 : 1757-67.

20. Rauch A, Legendre P, Christophe OD, et al. Antibody-based prevention of von Willebrand factor degradation mediated by circulatory assist devices. Thromb Haemost 2014 ; 112 : 1014-23.

21. Schiele F, van Ryn J, Canada K, et al. A specific antidote for dabigatran: functional and structural characterization. N Engl J Med 2013; 121 : 3554-62.

22. Pollack CV, Jr., Reilly PA, Eikelboom J, et al. Idarucizumab for dabigatran reversal. N Engl J Med 2015 ; 373 : 511-20.

23. Pollack CV, Jr., Reilly PA, van Ryn J, Idarucizumab for dabigatran reversal full cohort analysis. $2017 ; 377: 431-41$.

24. Godier A, Taylor G, Gaussem P. Inefficacy of platelet transfusion to reverse ticagrelor. N Engl J Med 2015 ; 372 : 196-7.

25. Buchanan $A$, Newton P, Pehrsson $S$, et al. Structural and functional characterization of a specific antidote for ticagrelor. Blood 2015; 125 : 3484-90.

26. Muczynski V, Casari C, Moreau F, et al. A factor VIII-nanobody fusion protein forming an ultrastable complex with VWF: effect on clearance and antibody formation. Blood 2018 ; 132 : 1193-7

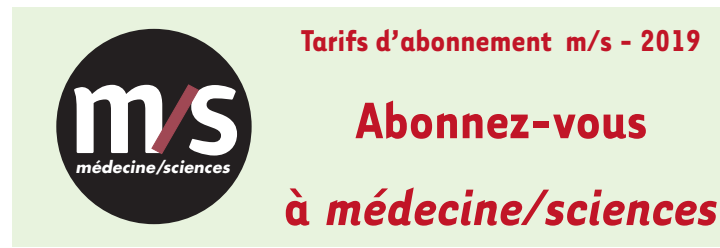

Tarifs d'abonnement $\mathrm{m} / \mathrm{s}-2019$

Abonnez-vous

à médecine/sciences
$>$ Grâce à $m / s$, vivez en direct les progrès des sciences biologiques et médicales

Bulletin d'abonnement page 1230 dans ce numéro de $\mathrm{m} / \mathrm{s}$
TIRÉS À PART

y. Gruel 